\title{
PROJETO PARA CONFIABILIDADE: APLICADO NO PROCESSO DE PROJETO DE EQUIPAMENTOS MÉDICOS
}

Beatriz Araujo Rodrigues (beatrizaraujorodrigues@gmail.com) - Programa de Pós-graduação em Sistema Mecatrônicos -Universidade de Brasília.

Suélia Rodrigues Fleury Rosa (rodrigues.suelia@gmail.com) - Programa de Pós-graduação em Engenharia Biomédica - Universidade de Brasília

Andrea Cristina dos Santos (andreakiek@gmail.com) - Programa de Pós-graduação em Sistema Mecatrônicos -Universidade de Brasília.

\section{RESUMO}

A confiabilidade do equipamento médico é um dos principais atributos exigidos pelos órgãos reguladores do sistema saúde, uma vez que o ambiente hospitalar apresenta características singulares. O processo de projeto de equipamentos médicos (PPEM) para ambientes hospitalares (como salas de cirurgias) deve observar, além dos requisitos dos clientes, os requisitos normativos legais de órgãos reguladores durante o projeto. O objetivo deste artigo é apresentar um conjunto de métodos, técnicas e modelos para confiabilidade que podem antecipar o atendimento dos requisitos normativos e legais. Para tanto, além da revisão da literatura sobre os requisitos normativos e legais de órgãos reguladores do sistema de saúde para equipamentos médicos de Classe III e IV, foi realizada uma revisão sistemática da literatura sobre o projeto para confiabilidade no PPEM. Por meio da análise da literatura foi possivel mapear os métodos e técnicas que podem ser aplicados para antecipar o atendimento requisitos normativo legais nas fases do PPEM. Adicionalmente foi verificado que não existe consenso sobre os métodos e técnicas sugeridos por diferentes autores. A literatura apresenta uma lacuna referente a indicação de métodos e técnicas específicos de projeto de confiabilidade para o PPEM.

Palavras chave: Confiabilidade; Equipamentos Médicos; Instrumentos Normativos; Projeto para Confiabilidade.

Área: Confiabilidade, Mantenabilidade e Disponibilidade

\section{INTRODUÇÃO}

A confiabilidade de um sistema é frequentemente definida como uma probabilidade de um sistema executar todas as suas funções pretendidas satisfatoriamente por um período específico de tempo e condições de uso (WEININGER; PECHT, 2009; RAHEJA; GULLO, 2012; SCHNEIDEWIND et al., 2010). O projeto para confiabilidade busca assegurar o atendimento dos requisitos de confiabilidade por meio do mapeamento de falhas das funções críticas do sistema nas fases do processo de projeto de produto (RAHEJA; GULLO, 2012).

O processo de projeto de equipamentos médicos é uma atividade complexa devido a sua relação direta com a saúde e vida dos pacientes. Falhas podem ser altamente dispendiosas em termos de fatalidades, lesões e perdas econômicas, fazendo com que as preocupações com eficácia, segurança e confiabilidade cresçam (DHILLON, 2000; MARTINI, 2005; CHENG; DAS; PECHT, 2011; LIN et al., 2014). Logo, quando se trata de equipamentos médicos, a falha não é uma opção, uma vez que pode ameaçar a vida do paciente dependendo do tipo de 
equipamento. Neste contexto, o processo de projeto de equipamentos médicos envolve o atendimento de múltiplos requisitos regulatórios e restrições impostos por agências regulamentadoras. Para que se possa registrar um equipamento médico e receber autorização para comercializa-lo é necessário que seja gerado um dossiê contendo dados sobre o desenvolvimento do equipamento contendo informações sobre a eficácia, segurança e e confiabilidade do equipamento.

Logo, todos os cenários de modo de falha devem ser investigados no PPEM com o objetivo de definir a melhor estratégia de projeto do equipamento, seja pela mudança nas suas funcionais, instalações de alarmes ou até mesmo instalações de redundâncias dependendo do tipo de equipamento.

O objetivo deste artigo é apresentar um conjunto de métodos, técnicas e modelos do projeto para confiabilidade que possam auxiliar o atendimento dos requisitos normativos e legais durante o Processo de Projeto de Equipamentos Médicos (PPEM).

Para tanto, além da revisão da literatura sobre os requisitos normativos e legais de órgãos reguladores do sistema de saúde para equipamentos de Classe III e IV foi realizada uma revisão sistemática da literatura sobre o Projeto para Confiabilidade (DfR - Design for Reliability) no Processo de Projeto de Equipamentos Médicos (PPEM).

\section{REQUISITOS NORMATIVOS E LEGAIS PARA EQUIPAMENTOS MÉDICOS}

Segundo King, Fries e Johnson (2014), o processo de projeto em engenharia biomédica envolve oportunidades sujeitas a restrições. Dentro desse contexto, as restrições envolvem: preocupações da sociedade no projeto de equipamentos médicos, como o atendimento de múltiplos requisitos regulatórios de agências reguladoras. Para tanto deve ser feita uma análise de confiabilidade no equipamento ao passo que o produto é desenvolvido.

Inicialmente fez-se um estudo dos requisitos normativos legais vigentes no Brasil que contemplam: Resoluções (RE), Resoluções de Diretoria Colegiada (RDC), Instruções Normativas (IN) e Normas Brasileiras (NBR) da Comissão Eletrotécnica Internacional (Internacional Electrotechnical Commission, IEC) e da Organização Internacional para Padronização (Internacional Organization for Standardization, ISO).

A pesquisa dos instrumentos normativos foi delimitada nos dispositivos do desenvolvimento de produtos para saúde de Classe de Risco III e IV (classificado pela RDC ${ }^{\circ}$ 185/01). Identificou-se 33 legislações aplicáveis diretamente. As legislações identificadas para esta classe de equipamento médico foram extraídas do repositório da Agência Nacional de Vigilância Sanitária (Anvisa), membro do Fórum Regulatório Internacional de Dispositivos Médicos (International Medical Device Regulatory Forum, IMDRF) no Brasil e da Associação Brasileira de Normas Técnicas (ABNT) (ANVISA, 2017; ABNT, 2017; CERETTI et al., 2016).

\section{REVISÃO SISTEMÁTICA DA LITERATURA}

A revisão sistemática foi desenvolvida para executar um amplo e consistente levantamento literário de aplicações de confiabilidade em equipamentos médicos. Este método de pesquisa utiliza a literatura como uma visualização de entrada para coletar todas as evidências que podem ser classificadas dentro de critérios de elegibilidade pré-determinados, a fim de responder a uma pergunta de pesquisa específica (HIGGINS; GREEN, 2011; PEGORARO; PAULA, 2017). 
Baseado nos argumentos da introdução, as questões de pesquisa foram: Quais são os principais métodos, ferramentas e fases aplicados no PPEM nos últimos dezesseis anos? Existe um consenso literário de aplicação de confiabilidade?

Fundamentado nessas questões, e foram estabelecidas as palavras-chave de pesquisa que caracterizam o primeiro filtro para seleção da pesquisa. As palavras-chave determinadas $a$ priori foram: "Medical Device Design for Reliability", "Medical System Reliability", "Electronics Systems Reliability", "Methodology of Mechatronic Design", "Test Reliability of Biomedical Devices", "Mechatronic Design", "Medical Devices Design", "Design of Biomedical Devices" e "Medical Devices Reliability" como termos de um eixo do referencial teórico.

Buscou-se utilizando essas palavras-chave na base Web of Science, textos publicados a partir do ano de 2006, sendo artigos de revista, livros, revisões de livros ou revisões para os tipos de documentos. A busca retornou uma massa de 181 referências. O segundo refinamento dos periódicos encontrados foi feito, nesta ordem, por título, por resumo, pela disponibilidade de acesso ao texto via periódico da CAPES, reduzindo a massa de artigos em 89,5\% com relação aos inicialmente encontrados, restando 19 artigos alinhados ao tema da pesquisa.

\subsection{Análise da Literatura}

Os fatores considerados na análise dos artigos foram: as fases do PPEM em que a confiabilidade foi considerada e o uso de ensaios e testes para análise da confiabilidade. Sendo que todos estes requisitos deveriam ser aplicados a um produto com fins médicos. Artigos que não possuíam nenhum destes dois fatores foram desconsiderados.

A definição das fases do PPEM foi baseada nos modelos de projeto para engenharia e equipamentos médicos expostos por El-Haik e Mekki (2011), Pietzsch et al. (2009), Ceretti et al. (2016), Ogrodnik (2012), Pahl e Beitz (2013), Pugh (1991) e Lantada (2013). A compilação e análise desses modelos resultou em seis fases do desenvolvimento para equipamentos médicos: Planejamento do Produto, Projeto Conceitual, Engenharia Básica, Projeto Detalhado, Produção e Venda e Manutenção.

A primeira análise nos artigos versa sobre a fase do desenvolvimento do produto em que a confiabilidade foi considerada, uma vez que o desenvolvimento de um equipamento médico confiável e seguro, considera a confiabilidade em todas as fases de desenvolvimento (CROWE; FEINBERG, 2001). Esta análise se mostra importante uma vez que verifica as fases do produto em que os pesquisadores dão maior atenção a confiabilidade e sua aplicabilidade.

Conforme ilustra a Tabela 1, a aplicação de confiabilidade é mais frequente nas fases de planejamento do produto (mencionada em 36,8\% dos artigos), engenharia básica (mencionada em 26,3\% dos artigos) e venda e manutenção (mencionada em 26,3\% dos artigos). Grande parte dos autores concentra a análise de confiabilidade em apenas uma fase do desenvolvimento, apenas Chiodo e Lauria (2015) dentre as literaturas pesquisadas utiliza confiabilidade em mais de uma fase do PPEM.

A segunda análise aborda os principais testes executados no equipamento, e suas características quanto ao processo de identificação da falha, ilustrado na Tabela 2.

Um programa de testes adequado é característica essencial para uma boa análise de confiabilidade e fornece a máxima quantidade de informações sobre falhas do equipamento (DHILLON, 2000; KING; FRIES; JOHNSON, 2014). Os dados de falha do dispositivo são um requisito essencial para a análise matemática de confiabilidade. 
Aproximadamente metade (42\%) dos autores que fazem a análise de confiabilidade no PPEM não utilizaram testes. Autores como Cauffriez et al. (2016); Kuecuekbalaban et al. (2014); Caffery, Burton e Richardson (2010) e Shah e Maisel (2006) lançaram mão de testes em sua análise de confiabilidade, embora usem dados de equipamentos na fase de uso e de normas para embasarem as estimativas.

As análises dos artigos estudados evidenciaram que há lacunas na aplicação de confiabilidade durante o PPEM, uma vez que não se identificou consenso quanto a fase de aplicação da confiabilidade no PPEM e quanto a utilização de testes para aferi-la.

Tabela 1. Aplicação de confiabilidade de acordo com as fases do desenvolvimento para produtos médicos. Fonte: Próprio autor.

\begin{tabular}{|c|c|c|c|}
\hline Fases do PPEM & Referências & $\begin{array}{l}\text { Quantidade } \\
\text { De Artigos }\end{array}$ & $\%$ \\
\hline $\begin{array}{l}\text { Planejamento do } \\
\text { Produto }\end{array}$ & $\begin{array}{l}\text { Keedy e Feng (2013); Kuecuekbalaban et al. (2014); Pang, } \\
\text { Lewis e Lee (2010); Lin et al. (2014); Caffery, Burton e } \\
\text { Richardson (2010); Mhenni et al. (2014); Andel et al. (2015) }\end{array}$ & 7 & 36,8 \\
\hline Projeto Conceitual & Abdali-Mohammadi, Bajalan e Fathi (2015) & 1 & 5,3 \\
\hline Engenharia Básica & $\begin{array}{l}\text { Habas et al. (2007); Ni e Broenick (2014); Sayil e Wang } \\
\text { (2014); Vogel, Auersperg e Michel (2009); Chiodo e Lauria } \\
\text { (2015) }\end{array}$ & 5 & 26,3 \\
\hline Projeto Detalhado & Giraldo-Cadavid et al. (2016) & 1 & 5,3 \\
\hline Produção & Chiodo e Lauria (2015) & 1 & 5,3 \\
\hline Venda e Manutenção & $\begin{array}{l}\text { Cauffriez et al. (2016); Fukuda (2006); Maisel et al. (2006); } \\
\text { Shah e Maisel (2006); Taghipour, Banjevic e Jardine (2011) }\end{array}$ & 5 & 26,3 \\
\hline
\end{tabular}

Tabela 2. Detalhamento dos testes executados nos produtos. Fonte: Próprio autor.

\begin{tabular}{|c|c|}
\hline Testes & Descrição \\
\hline Validação & $\begin{array}{l}\text { Processo de avaliação do sistema ou componente durante ou ao fim do processo de desenvolvimento } \\
\text { com a fim de determinar quais requisitos específicos são satisfeitos (Giraldo-Cadavid et al. (2016)) }\end{array}$ \\
\hline $\begin{array}{l}\text { Caixa } \\
\text { Preta }\end{array}$ & $\begin{array}{l}\text { Teste executado sem nenhum conhecimento da estrutura interna que verifica se os requisitos foram } \\
\text { extraídos do ponto de vista do usuário final (Cauffriez et al. (2016); Mhenni et al. (2014)) }\end{array}$ \\
\hline $\begin{array}{l}\text { Caixa } \\
\text { Branca }\end{array}$ & $\begin{array}{l}\text { Executado com conhecimento da estrutura interna do dispositivo do ponto de vista do desenvolvedor } \\
\text { (Cauffriez et al. (2016); Mhenni et al. (2014)) }\end{array}$ \\
\hline Software & $\begin{array}{l}\text { São vários níveis de avaliação, consistindo na interface entre o programador e o ambiente do } \\
\text { software (Habas et al. (2007); Caffery, Burton e Richardson (2010); Sayil e Wang (2014)) }\end{array}$ \\
\hline Funcional & $\begin{array}{l}\text { Verifica quais os requisitos funcionais têm sido satisfeitos (Abdali-Mohammadi, Bajalan e Fathi } \\
\text { (2015); Habas et al. (2007); Ni e Broenink (2014); Sayil e Wang (2014); Vogel, Auersperg e Michel } \\
(2009) \text { ) }\end{array}$ \\
\hline Robustez & $\begin{array}{l}\text { Feito para determinar como o software se comporta dadas entradas inesperadas (Keedy e Feng } \\
\text { (2013); Pang, Lewis e Lee (2010); Ni e Broenink (2014)) }\end{array}$ \\
\hline Segurança & $\begin{array}{l}\text { Verifica o desempenho do produto de maneira segura e que uma avaliação completa de segurança de } \\
\text { projeto tenha sido feita (Keedy e Feng (2013)) }\end{array}$ \\
\hline $\begin{array}{l}\text { Não } \\
\text { Utilizaram } \\
\text { Testes }\end{array}$ & $\begin{array}{l}\text { Não foi utilizado nenhum tipo de teste para comprovar a confiabilidade do produto (Chiodo e Lauria } \\
\text { (2015); Fukuda (2006); Kuecuekbalaban et al. (2014); Lin et al. (2014); Maisel et al. (2006); Shah e } \\
\text { Maisel (2006); Taghipour, Banjevic e Jardine (2011); Andel et al. (2015). }\end{array}$ \\
\hline
\end{tabular}




\section{O PROJETO PARA CONFIABILIDADE NO PPEM}

Observou-se que as orientações dos órgãos de regulamentação de equipamentos médicos no Brasil são orientadas em grande parte ao projeto de produtos farmacêuticos, deixando lacunas nas orientações referentes ao projeto de equipamentos médicos. Adicionalmente, embora a ANVISA exija apresentação das provas de segurança e confiabilidade do equipamento junto ao processo de registro do produto, os responsáveis pelo equipamento assumem perante a agência que a inobservância dos itens estabelecidos neste termo de responsabilidade constituirá uma infração sanitária, ficando sujeitos as penalidades previstas em lei.

Os registros nos órgãos de controle dos equipamentos médicos geralmente são indicados nos modelos para o processo de desenvolvimento de produtos/equipamentos nas fases finais (ElHaik; Mekki , 2011; Pietzsch et al., 2009; Ceretti et al., 2016; Ogrodnik, 2012; Pahl e Beitz, 2013; Lantada, 2013). Contudo, para executar os registros do equipamento na ANVISA, os dados e informações, com as provas de segurança e confiabilidade do equipamento serão gerados nas fases anteriores do PPEM.

Logo, com base na revisão sistemática da literatura infere-se que ainda existem lagunas na orientação do projeto para confiabilidade quando sua aplicação em equipamentos médicos, seja por uma literatura acadêmica ainda dispersa, ou orientações dos órgãos de regulamentação insipientes.

Considerando estes argumentos, foi proposta uma sistemática para aplicação da confiabilidade nas fases do PPEM, considerando os instrumentos normativos e legais apresentado na Tabela 3. Neste são apresentado os principais métodos, técnicas de projeto para confiabilidade ao longo do PPEM e os respectivos instrumentos normativos.

Para proporcionar confiabilidade a um produto médico seis etapas devem ser levadas em consideração: a Análise, os Testes, os Dados de Falha, as Métricas de Confiabilidade e as Áreas Associadas. A Análise compõe-se de três áreas inter-relacionadas: a análise de erros humanos, a análise das partes/componentes e a análise de software. A aplicação de cada uma dessas áreas tem como objetivo tornar o produto seguro e confiável.

\section{CONSIDERAÇÕES FINAIS}

A revisão sistemática permitiu identificar o emprego do projeto para confiabilidade no PPEM nos últimos dez anos indicando as principais lacunas na área. Resultado da revisão sistemática da literatura buscou-se definir quais eram as principais fases do PPEM, suas atividades e abordagem de confiabilidade aplicada nas diferentes fases. Mediante a revisão dos requisitos normativos e legais aplicados no Brasil, pelos órgãos regulamentadores, como Agência Nacional de Vigilância Sanitária (ANVISA) e Associação Brasileira de Normas Técnicas (ABNT) mapeou-se as normas relacionadas a equipamentos médicos.

O mapeamento das normas relacionadas a equipamentos médicos, para equipamentos de Classe III e IV, em relação a literatura de confiabilidade nas fases do PPME, ilustra de forma didática o sequenciamento das atividades, a sua inter-relação com o momento de aplicação dos normativos legais a serem consultadas durante o projeto. Ou seja, permite visualizar a necessidade de atendimento dos requisitos legais brasileiros.

Através da revisão sistemática, verificou-se que (i) os artigos concentram a aplicação da confiabilidade em apenas uma das fases do PPEM (ii) não existe consenso na literatura quanto a aplicação do projeto para confiabilidade no PPEM e (iii) não existe um entendimento entre os autores pesquisados sobre utilizar testes ou não para aferir a confiabilidade. 
Com base nas informações levantadas na revisão da literatura sistemática, os próximos passos desta pesquisa envolvem o projeto de um equipamento médico com ênfase no projeto para confiabilidade.

Tabela 3 - Caracterização da confiabilidade segundo as fases do desenvolvimento e normas associadas.

\begin{tabular}{|c|c|c|c|c|}
\hline $\begin{array}{c}\text { Fase do } \\
\text { PPEM }\end{array}$ & Atividades & $\begin{array}{l}\text { Abordagem } \\
\text { Confiabilidade }\end{array}$ & Métodos, Técnicas e Modelos & Legislações \\
\hline 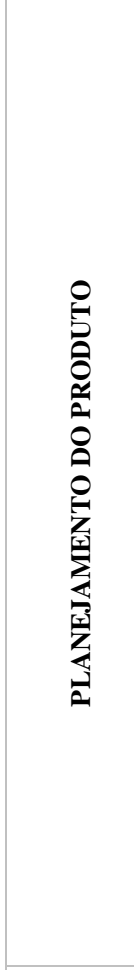 & $\begin{array}{l}\text { Verifica o estado da } \\
\quad \text { arte } \\
\text { •Executa o } \\
\text { Benchmarking } \\
\text { •Define as } \\
\text { necessidades } \\
\text { •Estabelece } \\
\text { requisitos } \\
\text { (especificações, } \\
\text { tarefas a serem } \\
\text { feitas) } \\
\text { • Estima custos } \\
\text { Estabelece a agenda } \\
\text { total e data de } \\
\text { lançamento }\end{array}$ & $\begin{array}{l}\text { Garantia } \\
\text { Qualidade, } \\
\text { Garantia da } \\
\text { Segurança }\end{array}$ & $\begin{array}{c}\text { Qualidade (Diagrama de Pareto, } \\
\text { Diagrama de Causa e Efeito, Diagrama } \\
\text { de Dispersão, Falhas de Verificação, } \\
\text { Histograma, Fluxograma, QFD, } \\
\text { Gráficos de Controle) } \\
\text { Segurança (Análise de risco preliminar, } \\
\text { Técnica de Revisão de Operações, } \\
\text { Análise de Erro Humano, Análise de } \\
\text { Risco Operacional, Análise da Árvore } \\
\text { de Falhas (FTA, Fault Tree Analysis), } \\
\text { Análise do Modo de Falha e Efeitos } \\
\text { (FMEA, Failure Mode and Effects } \\
\text { Analysis), Revisão de Riscos e } \\
\text { Operacionabilidade, Análise da Causa } \\
\text { Raiz) }\end{array}$ & 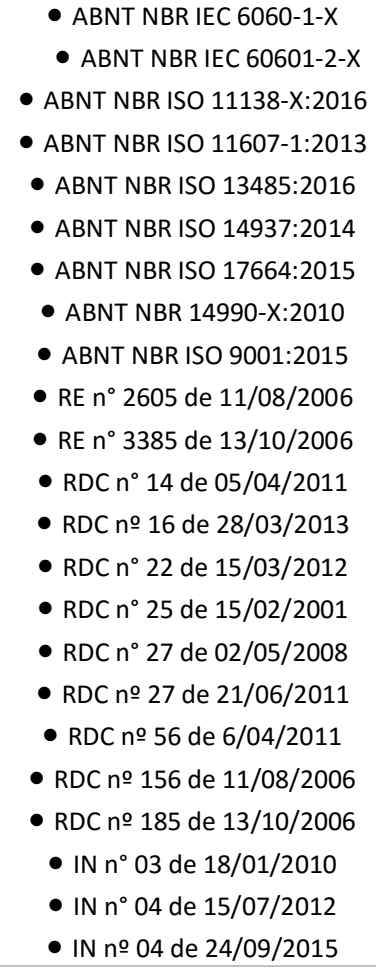 \\
\hline 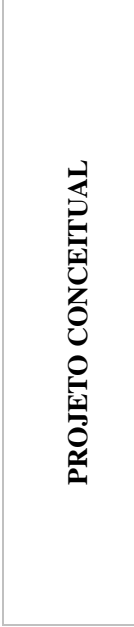 & $\begin{array}{l}\text { a a análise funcional } \\
\text { •Formalização dos } \\
\text { requisitos } \\
\text { funcionais e } \\
\text { restrições } \\
\text { •Elabora a matriz } \\
\text { morfológica } \\
\text { •Cria a arquitetura } \\
\text { inicial }\end{array}$ & $\begin{array}{c}\text { Análise De Erros } \\
\text { Humanos, } \\
\text { Garantia da } \\
\text { Segurança }\end{array}$ & $\begin{array}{c}\text { Análise de erros humanos (FMEA, } \\
\text { Método da Taxa de Transmissão, } \\
\text { Método da Árvore de Probabilidade, } \\
\text { FTA) } \\
\text { Segurança (Análise de risco preliminar, } \\
\text { Técnica de Revisão de Operações, } \\
\text { Análise de erro humano, Análise de } \\
\text { Risco Operacional, Revisão de Riscos } \\
\text { e Operacionalidade, Análise da Causa } \\
\text { Raiz) }\end{array}$ & $\begin{array}{l}\text { - ABNT NBR IEC 6060-1-X } \\
\text { - ABNT NBR IEC 60601-2-X } \\
\text { - ABNT ISO/TS 19218-X:2014 } \\
\text { - ABNT NBR ISO 11138-X:2016 } \\
\text { - ABNT NBR ISO 13485:2016 } \\
\text { - ABNT NBR ISO 17664:2015 } \\
\text { - ABNT NBR 14990-X:2010 } \\
\text { - ABNT NBR ISO 15223-1:2015 } \\
\text { - ABNT NBR 16328:2014 } \\
\text { - RDC no } 10 \text { de 20/02/2015 } \\
\text { RDC no } 15 \text { de 28/03/2014 } \\
\text { - RDC no } 27 \text { de 21/06/2011 } \\
\text { - RDC n } 33 \text { de 14/06/2012 } \\
\text { RDC no } 56 \text { de 06/04/2001 } \\
\text { - RDC no } 156 \text { de 11/08/2006 }\end{array}$ \\
\hline
\end{tabular}




\begin{tabular}{|c|c|c|c|c|}
\hline 感 & $\begin{array}{l}\text { •Define a forma e } \\
\text { geometria } \\
\text { •Realiza a seleção } \\
\text { do material } \\
\text { •Estabelece o } \\
\text { processo de } \\
\text { seleção preliminar } \\
\text { da manufatura } \\
\text { •Revisão e } \\
\text { atualização da } \\
\text { avaliação técnica } \\
\text { e financeira } \\
\text { •Executa testes de } \\
\text { verificação }\end{array}$ & $\begin{array}{l}\text { Análise de } \\
\text { Software, Análise } \\
\text { das } \\
\text { Partes/Component } \\
\text { es, Custos, } \\
\text { Garantia da } \\
\text { Segurança, Testes } \\
\text { para } \\
\text { Confiabilidade }\end{array}$ & $\begin{array}{l}\text { Análise de Software (Complexidade de } \\
\text { MacCabe, Medidas de Halstead, Tamanho } \\
\text { do Programa, Vocabulário do Software, } \\
\text { Volume do Software, Volume Potencial) } \\
\text { Análise das partes/componentes (FMEA, } \\
\text { FTA, Taxa de falha, Markov, Falha de } \\
\text { Causa Comum) } \\
\text { Custos (Estimação de custo FMEA, } \\
\text { Estimação de custo modelagem/alocação de } \\
\text { confiabilidade, Estimação de custo de teste } \\
\text { de confiabilidade, Estimação de custo da } \\
\text { previsão de confiabilidade, Estimação de } \\
\text { custo de manutenção de software, } \\
\text { Estimação de custo do desenvolvimento de } \\
\text { software, Capacidade de Custo) } \\
\text { Segurança (Análise de Risco Preliminar, } \\
\text { Técnica de Revisão de Operações, Análise } \\
\text { de Erro Humano, Análise de Risco } \\
\text { Operacional, Revisão de Riscos e } \\
\text { Operacionabilidade, Análise da Causa Raiz) } \\
\text { Testes para confiabilidade (Teste } \\
\text { Ambiental, Teste De Estresse, Teste De } \\
\text { Evento, Do Tempo, Teste De Erro, Teste } \\
\text { De Segurança, Teste Funcional, Teste Da } \\
\text { Caixa Branca, Teste De Forma Livre, Teste } \\
\text { de Acompanhamento de Falhas (FRACAS, } \\
\text { Test Failure Tracking)) }\end{array}$ & 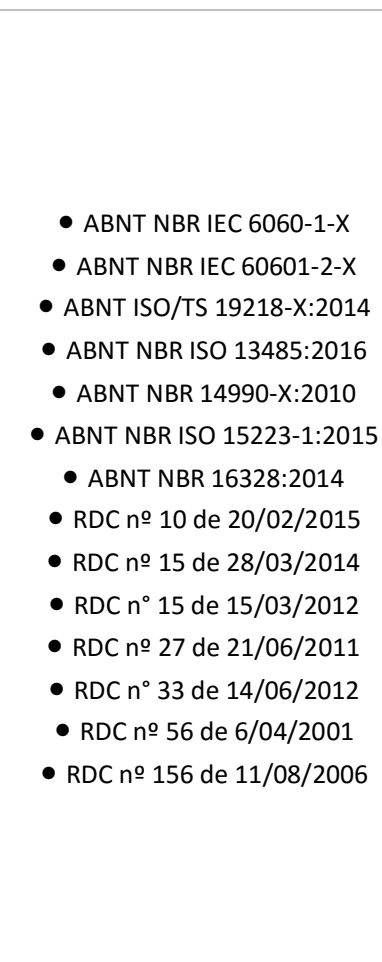 \\
\hline 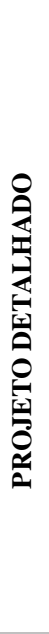 & $\begin{array}{c}\text { •Realiza a seleção } \\
\text { da manufatura } \\
\text { •Escolha e } \\
\text { atualização do } \\
\text { desenho final } \\
\text { •Simulação de } \\
\text { processos para } \\
\text { riscos e melhorias } \\
\text { Conferências finais } \\
\text { •Revisão dos dados } \\
\text { de falha testados e } \\
\text { em campo }\end{array}$ & $\begin{array}{l}\text { Avaliação de } \\
\text { Risco, Análise dos } \\
\text { Dados de Falha, } \\
\text { Garantia da } \\
\text { Segurança }\end{array}$ & $\begin{array}{c}\text { Avaliação de risco (FMEA, Estudo de } \\
\text { Operacionabilidade e Risco (HAZOP, } \\
\text { Hazard and Operability Study), Análise } \\
\text { de Árvore de Evento) } \\
\text { Análise dos dados de falha (Bartlett e } \\
\text { Estimativa de Máxima Verossimilhança) } \\
\text { Segurança (Análise de Risco Preliminar, } \\
\text { Técnica de Revisão de Operações, Análise } \\
\text { de Erro Humano, Análise de Risco } \\
\text { Operacional, Revisão de Riscos e } \\
\text { Operacionabilidade, Análise da Causa } \\
\text { Raiz) }\end{array}$ & $\begin{array}{l}\text { - ABNT NBR IEC 6060-1-X } \\
\text { - ABNT NBR IEC 60601-2-X } \\
\text { - ABNT ISO/TS 19218-X:2014 } \\
\text { - ABNT NBR ISO 11138-X:2016 } \\
\text { - ABNT NBR ISO 11607-1:2013 } \\
\text { - ABNT NBR 14990-X:2010 } \\
\text { - ABNT NBR ISO 13485:2016 } \\
\text { - ABNT NBR 16328:2014 } \\
\text { - RDC no } 10 \text { de 20/02/2015 } \\
\text { - RDC no } 15 \text { de 28/03/2014 } \\
\text { - RDC n } 15 \text { de 15/03/2012 } \\
\text { - RDC no } 27 \text { de 21/06/2011 } \\
\text { - RDC n } 33 \text { de 14/06/2012 } \\
\text { - RDC no } 56 \text { de 06/04/2001 } \\
\text { - IN nº } 04 \text { de 15/07/2012 }\end{array}$ \\
\hline 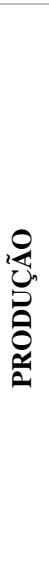 & $\begin{array}{l}\text { •Construção do } \\
\text { primeiro protótipo } \\
\text { 'Término do manual } \\
\text { e detalhamento } \\
\text { das especificações } \\
\text { •Testes de uso } \\
\text {-Início da produção }\end{array}$ & $\begin{array}{l}\text { Avaliação de } \\
\text { Risco, Custos, } \\
\text { Análise de } \\
\text { Confiabilidade }\end{array}$ & $\begin{array}{l}\text { Avaliação de risco (FMEA, Estudo de } \\
\text { operacionabilidade e risco (HAZOP, } \\
\text { Hazard and Operability Study) } \\
\text { Custos (Estimação de custo FMEA, } \\
\text { Estimação de custo modelagem/alocação } \\
\text { de confiabilidade, Estimação de custo de } \\
\text { teste de confiabilidade, Estimação de } \\
\text { custo da previsão de confiabilidade, } \\
\text { Estimação de custo de manutenção de } \\
\text { software, Estimação de custo do } \\
\text { desenvolvimento de software, Capacidade } \\
\text { de Custo) } \\
\text { Análise de Confiabilidade (Curva da } \\
\text { banheira, Confiabilidade, Tempo Médio } \\
\text { entre Falhas (MTBF, Mean Time Between } \\
\text { Failures), Taxa de Risco, Taxa de Falhas) }\end{array}$ & 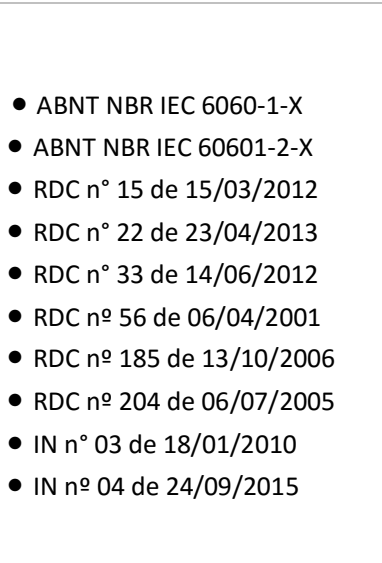 \\
\hline
\end{tabular}




\section{REFERÊNCIAS}

ABDALI-MOHAMMADI, F.; BAJALAN, V.; FATHI, A. Toward a fault tolerant architecture for vital medical-based wearable computing. Journal of medical systems, Springer, v. 39, n. 12, p. 149, 2015.

ABNT. Associação Brasileira de Normas Técnicas - Catálogo de Normas Produtos para Saúde. Disponível em: < http://www.abnt.org.br/normalizacao/abnt-catalogo >. Acesso em 07 de abril de 2017.

ANDEL, J. V. et al. What makes a good home-based nocturnal seizure detector? a value sensitive design. PloS one, Public Library of Science, v. 10, n. 4, p. e0121446, 2015.

ANVISA. Agência Nacional de Vigilância Sanitária - Legislação de Produtos Médicos. Disponível em:< http://portal.anvisa.gov.br/legislacao\#/>. Acesso em: 04 de abril de 2017.

CAFFERY, F. M.; BURTON, J.; RICHARDSON, I. Risk management capability model for the development of medical device software. Software Quality Journal, Springer, v. 18, n. 1, p. 81, 2010.

CAUFFRIEZ, L. et al. Bond graph modeling for fault detection and isolation of a train door mechatronic system. Control Engineering Practice, Elsevier, v. 49, p. 212-224, 2016.

CERETTI, E. et al. (Ed.). Biomedical Devices: Design, Prototyping, and Manufacturing. John Wiley \& Sons, 2016.

CHENG, S.; DAS, D.; PECHT, M. G. Using Failure Modes, Mechanisms, and Effects Analysis in Medical Device Adverse Event Investigations. In: ICBO. 2011.

CHIODO, E.; LAURIA, D. Some basic properties of the failure rate of redundant reliability systems in industrial electronics applications. IEEE Transactions on Industrial

Electronics, IEEE, v. 62, n. 8, p. 5055-5062, 2015.

CROWE, D.; FEINBERG, A. (Ed.). Design for reliability. CRC press, 2001.

DHILLON, B. S. Medical device reliability and associated areas. CRC Press, 2000.

EL-HAIK, B.; MEKKI, K. S. Medical device design for six sigma: A road map for safety and effectiveness. John Wiley \& Sons, 2011.

KING, P. H.; FRIES, R. C.; JOHNSON, A. T. Design of biomedical devices and systems. [S.1.]: CRC Press, 2014.

FUKUDA, M. Optical source reliability in recent optical fiber transmission systems and consumer electronics. Microelectronics Reliability, Elsevier, v. 46, n. 2, p. 263-269, 2006.

GIRALDO-CADAVID, L. F. et al. Design, development and validation of a new laryngopharyngeal endoscopic esthesiometer and range-finder based on the assessment of air-pulse variability determinants. Biomedical engineering online, BioMed Central, v. 15, n. 1, p. 52, 2016.

HABAS, P. A. et al. Reliability analysis framework for computer-assisted medical decision systems. Medical physics, Wiley Online Library, v. 34, n. 2, p. 763-772, 2007.

HIGGINS, J. PT; GREEN, S. (Ed.). Cochrane handbook for systematic reviews of interventions. John Wiley \& Sons, 2011.

JUNIOR, O. C.; OKUMURA, M. L. M.; YOUNG, R. I. M. The Application of an Integrated Product Development Process to the Design of Medical Equipment. In: Concurrent Engineering in the 21 st Century. Springer International 
KEEDY, E.; FENG, Q. Reliability analysis and customized preventive maintenance policies for stents with stochastic dependent competing risk processes. IEEE Transactions on reliability, v. 62, n. 4, p. 887-897, 2013.

KUECUEKBALABAN, P. et al. Exploring risks and benefits of point-of-care tests for healthcare and self-tests for laypersons: An interview study assessing complementary expert perspectives on diagnostic lab-on-a-chip systems. Technology and Health Care, v. 22, n. 6, p. 817-833, 2014.

LANTADA, A. D. (Ed.). Handbook on advanced design and manufacturing technologies for biomedical devices. Springer, 2013.

LIN, Q. et al. Human reliability assessment for medical devices based on failure mode and effects analysis and fuzzy linguistic theory. Safety science, v. 62, p. 248-256, 2014.

MAISEL, W. H. et al. Pacemaker and icd generator malfunctions: analysis of food and drug administration annual reports. Jama, American Medical Association, v. 295, n. 16, p. 19011906, 2006.

MARTINI, F. P. Práticas de manutenção em equipamentos de diagnóstico e terapia em serviços de oftalmologia na cidade de Porto Alegre. 2005.

MHENNI, F. et al. A sysml-based methodology for mechatronic systems architectural design. Advanced Engineering Informatics, Elsevier, v. 28, n. 3, p. 218-231, 2014.

NI, Y.; BROENINK, J. F. A co-modelling method for solving incompatibilities during codesign of mechatronic devices. Advanced engineering informatics, Elsevier, v. 28, n. 3, p. 232-240, 2014.

OGRODNIK, P. J. Medical device design: innovation from concept to market. Academic Press, 2012.

PAHL, G.; BEITZ, W. Engineering design: a systematic approach. Springer Science \& Business Media, 2013.

PANG, C. K.; LEWIS, F. L.; LEE, T. H. Modal parametric identification of flexible mechanical structures in mechatronic systems. Transactions of the Institute of Measurement and Control, Sage Publications Sage UK: London, England, v. 32, n. 2, p. 137-154, 2010.

PEGORARO, C.; PAULA, I. C. Requirements processing for building design: a systematic review. Production, v. 27, 2017.

PIETZSCH, J. B. et al. Stage-gate process for the development of medical devices. Journal of Medical Devices, v. 3, n. 2, p. 021004, 2009.

PUGH, S. Total design: integrated methods for successful product engineering. AddisonWesley, 1991.

RAHEJA, D. G.; GULLO, L. J. (Ed.). Design for reliability. John Wiley \& Sons, 2012.

SAYIL, S.; WANG, J. Coupling induced soft error mechanisms in nanoscale cmos technologies. Analog Integrated Circuits and Signal Processing, Springer, v. 79, n. 1, p. 115126, 2014.

SCHNEIDEWIND, N. et al. IEEE Reliability Society Technical Operations Annual Technical Report for 2010. IEEE Transactions on Reliability, v. 59, n. 3, p. 449-482, 2010.

SHAH, J. S.; MAISEL, W. H. Recalls and safety alerts affecting automated external defibrillators. JAMA, American Medical Association, v. 296, n. 6, p. 655-660, 2006. 
TAGHIPOUR, S.; BANJEVIC, D.; JARDINE, A. K. Reliability analysis of maintenance data for complex medical devices. Quality and Reliability Engineering International, Wiley Online Library, v. 27, n. 1, p. 71-84, 2011.

VOGEL, D.; AUERSPERG, J.; MICHEL, B. Advanced experimental and simulation approaches to meet reliability challenges of new electronics systems. Advanced Engineering Materials, Wiley Online Library, v. 11, n. 4, p. 309-315, 2009.

WOO, S. Reliability Design of Mechanical Systems: A Guide for Mechanical and Civil Engineers. Springer, 2017. 